\title{
Enhanced Retention of Chemotactic Bacteria in a Pore Network with Residual NAPL Contamination
}

\section{- Supporting Information}

Xiaopu Wang ${ }^{\S, \dagger}$, Larry M. Lanning ${ }^{\dagger}$ and Roseanne M. Ford ${ }^{\dagger}, *$

$\S$ School of Petroleum Engineering, China University of Petroleum, Qingdao, Shandong, China 266580

$\dagger$ Department of Chemical Engineering, School of Engineering and Applied Science,

University of Virginia, Charlottesville, VA, USA 22904

*Corresponding author phone: +1-434-924-6283; email: rmf3f@virginia.edu

Running title:

Chemotaxis in heterogeneous porous microfluidics

Keywords:

Chemotaxis, Pseudomonas putida, heterogeneity, porous media, microfluidics, bioremediation, biodegradation

Contact grant sponsor: NSF, the National Basic Research Program of China, National Natural Science Foundation of China

Contact grant number: EAR-0711377, EAR-1141400, 2015CB251200 and 51509260 


\section{NAPL Trapping}

As shown in Figure A1 as a side view of Figure 1d, a thin layer of water surrounds the toluene droplet, and the toluene droplet exhibits a high surface tension at the top openings, which generates an extra downward force $\mathrm{F}_{\mathrm{S}}$ on the droplet. Moreover, the glass surface on top also provides a downward resistance force $\mathrm{F}_{\mathrm{W}}$ on the toluene droplet, and both forces prevent the organic compound from being washed out. Thus, even though toluene has a lower density than water, toluene remains as a droplet in the bottom layer of the device.

Moreover, because the glass wall is hydrophilic, the hydrophobic toluene does not attach to the smooth glass surface; in other words, the water layer between the toluene droplet and the glass wall provides a conduit through which the water flow can always bypass the NAPL component. But because the NAPL reservoir has more spaces for the water to go around than the narrow pore channel, the pressure that can wash out the toluene in the pore channels is reduced at the reservoirs and is not enough to push out the toluene droplets. 


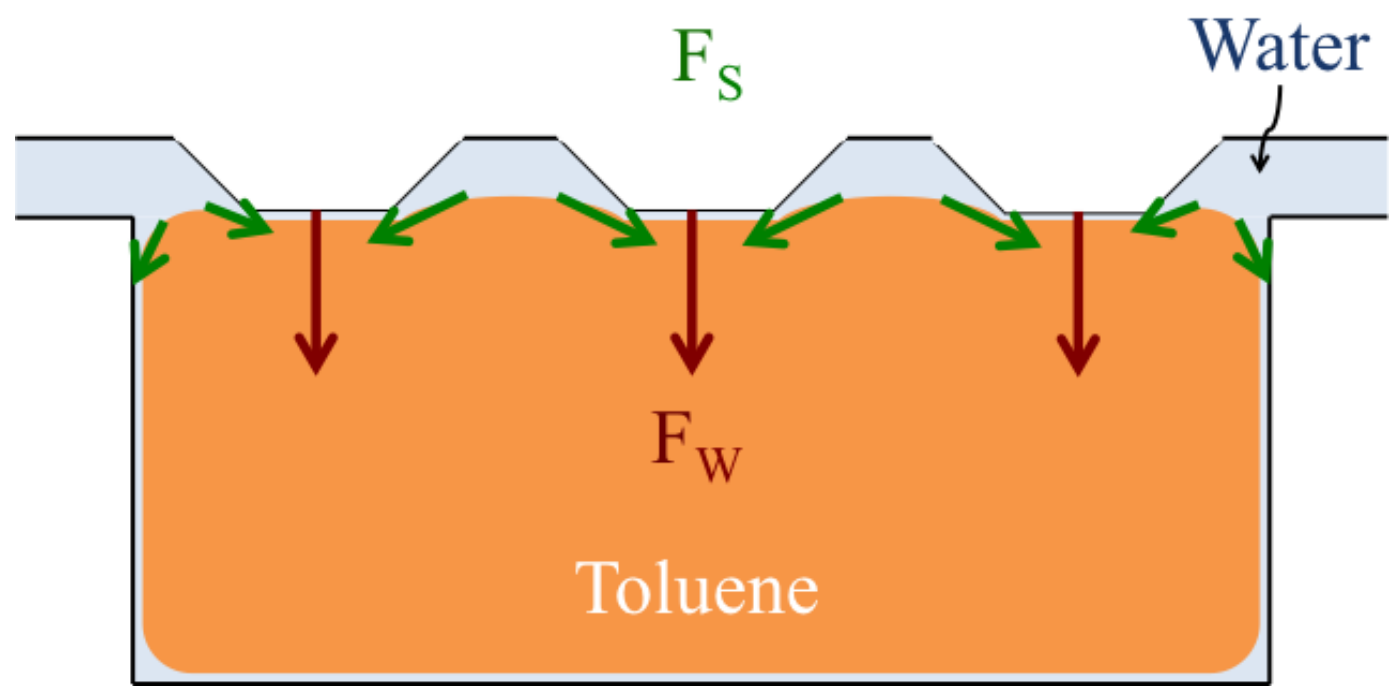

Figure A1. Schematic illustration of toluene entrapment in the microfluidic device. $F_{s}$ stands for the force vector induced from surface tension on a toluene droplet, and $\mathrm{F}_{\mathrm{w}}$ represents the resistance force from the glass wall.

After toluene droplets were trapped within the circular spaces of the device, a high flow rate was used to test their stability. The toluene droplets remained stable under injection rates comparable to $100 \mathrm{~m} / \mathrm{d}$ in the main channel and after 24 hours of continuous flow at $5 \mathrm{~m} / \mathrm{d}$. The stability resulted from the low solubility and high surface tension between toluene and water in the glass channel. Bacteria were transported into the fine pore network under high advective velocities in the main channel.

\section{FM 4-64 vs. Bacterial Motility}

An unfortunate consequence of the FM 4-64 staining was that although the membrane dye itself did not alter the bacterial properties, the excitation of fluorescence appeared to interfere with bacterial motility. The reason was likely because the FM 4-64 dye was linked to the plasma membrane, and the fluorescence excitation generated an extremely high level of energy absorbance and energy release in a very short time, which could 
reduce or diminish the membrane potential. Although no evidence indicated that the high-energy release killed the cell, the bacterial flagella could be greatly impacted as they are powered by the membrane potential. As a result, the fluorescence intensity could not be monitored in real time inside the pore network or the macrochannel of the microfluidic device. Instead, we recorded fluorescent intensity in real time at the outlet of the microfluidic device, so that the excitation would not affect the swimming bacteria within the pore network or macrochannel. The dimension of the affected zone was determined by the arc lamp precision and objective lens, and it could be directly measured from the bright area covered by the focused light beam. As shown in Figure A2, the affected zone (the green circle) has a diameter of about 2-3 mm, and it was far away from the main channel and pore network. Although there was scattered energy from the arc lamp beam all over the device, its intensity was much weaker than in the focused affected zone. Note that bacterial movement in the main channel and porous network was actually examined in bright field, and under those conditions the motility was not affected at all.

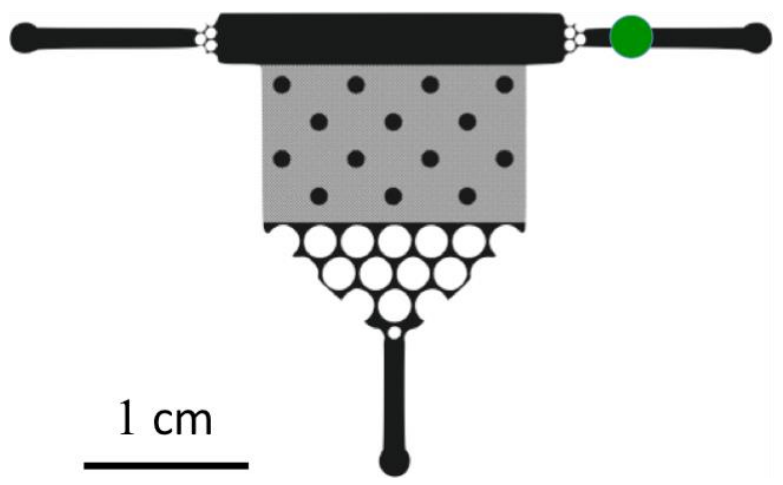

Figure A2. Affected zone (the green circle) by arc lamp light beam on the microfluidic device. 


\section{References}

1. Atencia, J.; Cooksey, G. A.; Locascio, L. E. A robust diffusion-based gradient generator for dynamic cell assays. Lab Chip. 2012, 12: 309-316

2. Ahmed, T.; Stocker, R. Experimental verification of the behavioral foundation of bacterial transport parameters using microfluidics. Biophys. J. 2008, 95(9): 44814493. 